\title{
On the relation between possibilistic logic and modal logics of belief and knowledge
}

\author{
Mohua Banerjee $^{a}$, Didier Dubois ${ }^{b}$, Lluis Godo $^{c}$ and Henri Prade ${ }^{b}$ \\ ${ }^{a}$ Dept. of Mathematics and Statistics, Indian Institute of Technology, Kanpur, India; \\ ${ }^{b}$ IRIT-CNRS, Université Paul Sabatier, Toulouse, France; \\ ${ }^{c}$ IIIA-CSIC, Bellaterra, Spain
}

\begin{abstract}
Possibility theory and modal logic are two knowledge representation frameworks that share some common features, such as the duality between possibility and necessity, as well as some obvious differences since possibility theory is graded but is not primarily a logical setting. In the last thirty years there have been a series of attempts, reviewed in this paper, for bridging the two frameworks in one way or another. Possibility theory relies on possibility distributions and modal logic on accessibility relations, at the semantic level. Beyond the observation that many properties of possibility theory have qualitative counterparts in terms of axioms of well-known modal logic systems, the first works have looked for (graded) accessibility relations that can account for the behavior of possibility and necessity measures. More recently, another view has emerged from the study of logics of incomplete information, which is no longer based on general Kripke-like models. On the one hand, possibilistic logic, closely related to possibility theory, mainly handles beliefs having various strengths. On the other hand, in the so-called meta-epistemic logic (MEL) an agent can express both all-or-nothing beliefs and explicitly ignored facts, by only using modal formulas of depth 1, and no objective ones; its semantics is based on subsets of interpretations viewed as epistemic states. The system $\mathrm{MEL}^{+}$is a KD45-like extension of MEL with objective formulas. Generalized possibilistic logic (GPL) extends both possibilistic logic and MEL, and has a semantics in terms of sets of possibility distributions. After a survey of these different attempts, the paper presents $\mathrm{GPL}^{+}$, a graded counterpart of $\mathrm{MEL}^{+}$that extends both MEL and GPL by allowing objective (sub)formulas. The axioms of $\mathrm{GPL}^{+}$are graded counterparts of those of $\mathrm{MEL}^{+}$, the semantics being based on pairs made of an interpretation (representing the real state of facts) and a possibility distribution (representing an epistemic state). S5-like extensions
\end{abstract}


of $\mathrm{MEL}^{+}$and $\mathrm{GPL}^{+}$, called $\mathrm{MEL}^{++}$and $\mathrm{GPL}^{++}$respectively, are also considered. Soundness and completeness of $\mathrm{GPL}^{+}$and and $\mathrm{GPL}^{++}$ are established. The paper also discusses the difference between MEL and S5 used as a standard epistemic logic, or used as a logic for rough sets that accounts for indiscernibility rather than incomplete information. We highlight the square of opposition as a common structure underlying modal logic, possibility theory, and rough set theory.

Keywords Modal logic, possibility theory, epistemic logic, rough sets

\section{Introduction}

Possibility theory has been originally introduced by Zadeh (1978a) as a framework for representing the uncertainty conveyed by linguistic statements. It is based on the notion of possibility distribution $\pi$, from which a maxitive possibility measure $\Pi(A)$ is defined as a consistency degree between this distribution representing the available information and the considered event $A$. This proposal is formally similar to, although fully independent of, the one previously developed in economics by Shackle (1961) based on the notion of degree of surprise (which corresponds to impossibility).

Although possibility theory has been the basis of an original approximate reasoning theory (Zadeh, 1979a), Zadeh's approach does not provide a logical setting, strictly speaking. It is only later, in the 1980's, that possibilistic logic, a logic that uses classical formulas associated with certainty levels (thought of as lower bounds of a necessity measure), has emerged (see (Dubois \& Prade, 2004, 2014) for introductions and overviews). Still, in the setting of his representation language PRUF, Zadeh (1978b) discusses the representation of statements of the form " $X$ is $A$ " (meaning that the possible values of the single-valued variable $X$ are fuzzily restricted by fuzzy set A) linguistically qualified in terms of truth, probability, or possibility. Interestingly enough, the representation of possibility-qualified statements led to possibility distributions over possibility distributions, but certainty-qualified statements were not considered at all, just because necessity measures (first proposed by Dubois \& Prade (1980)) as dual of possibility measures basically do not appear in Zadeh's works (with the exception of half a page in (Zadeh, 1979b)). Certainty-qualified statements were first considered in (Prade, 1985), and rediscussed in (Dubois \& Prade, 1990) in relation with two resolution principles (respectively inferring from two certainty-qualified propositions, and one certainty-qualified proposition together with a possi- 
bility qualified proposition). Their formal analogy with the inference rules existing in modal logic was stressed.

Such an analogy between possibility theory functions (including necessity measures) and modalities in modal logic was not coming as a surprise since the parallels between $N(A)=1-\Pi(A)$ and $\square p \leftrightarrow \neg \diamond \neg p$ (duality between necessity and possibility), between $N(A) \leq \Pi(A)$ and $\diamond p \rightarrow \square p$ (axiom $\mathrm{D}$ in modal logic systems), or between the characteristic axiom of necessity measures $N(A \cap B)=\min (N(A), N(B))$ and adjunction $(\square p \wedge \square q) \leftrightarrow \square(p \wedge q)$ (a theorem valid in modal system $\mathrm{K}$ ) had been already noticed. Nevertheless, no formal connection between modal logic and possibility theory existed in the early days, even if the idea of graded accessibility relations had been already proposed independently (Lakoff, 1973; Schotch, 1975) some years before the invention of possibility theory.

The striking parallel between possibility theory and modal logic eventually led to proposals for a modal analysis and encoding of possibility theory, one of which by Fariñas del Cerro \& Herzig (1991), later by Boutilier (1994), then extended to multiple-valued propositions (Hájek et al., 1994). Another more semantically-oriented trend was to build particular accessibility relations (Dubois et al., 1988; Harmanec \& Klir, 1994b) agreeing with possibility theory. The work in (Liau \& Fan, 2005; Liau \& Lin, 1992, 1993a,b) is also worth-mentioning in that respect.

Rather than putting possibility theory under the umbrella of (graded) modal logics, a quite different view has finally emerged by designing a logical system closer to classical logic, while still capable of handling simple certainty- or possibility-qualified statements. This type of epistemic logic is just a two-tiered propositional logic (an idea that first appears in (Dubois \& Prade, 2007)) where only propositional combinations of modal formulas of depth 1 can be handled. The resulting logic called MEL, ${ }^{1}$ where necessity and possibility are binary-valued, proved to be equivalent to a fragment of the normal modal logic system KD (Banerjee \& Dubois, 2009, 2014). MEL can be extended to graded modalities, thus extending possibilistic logic (Dubois et al., 1994; Lang et al., 1991) (where only conjunctions of certainty- or possibility-qualified statements are allowed) to a generalized possibilistic logic (GPL) (Dubois et al., 2012), where negation and disjunctions of weighted formulas are allowed. The semantics of MEL (resp. GPL) is no longer expressed by means of an accessibility relation, but in terms of a set of sets of models (resp. a set of possibility distributions), which agrees

\footnotetext{
${ }^{1}$ Originally standing for meta-epistemic logic (Banerjee \& Dubois, 2009) then minimal epistemic logic (Banerjee \& Dubois, 2014).
} 
with Zadeh's original semantical view of possibility-qualified statements.

MEL has been more recently extended to the handling of propositional combinations of objective formulas and modal formulas of depth 1 in the style of KD45, which gives the logical system MEL ${ }^{+}$(Banerjee et al., 2014). These mixed formulas are then semantically evaluated by pairs made of one interpretation (representing the real state of facts) and a non-empty set of interpretations (representing an epistemic state). The axioms of $\mathrm{MEL}^{+}$are those of MEL, while an S5-like version called $\mathrm{MEL}^{++}$also includes axiom T $(\square p \rightarrow p) . \mathrm{MEL}^{+}$and $\mathrm{MEL}^{++}$are respectively equivalent to the non-nested fragments of modal systems KD45 and S5.

The purpose of this paper is to briefly survey the literature on connections between modal logic and possibility theory, to compare the semantics of MEL to the semantics of epistemic logic and rough set logics, and to extend $\mathrm{MEL}^{+}$and $\mathrm{MEL}^{++}$with graded modalities, leading to corresponding extensions of GPL, called $\mathrm{GPL}^{+}$and $\mathrm{GPL}^{++}$respectively.

The paper is structured as follows. The next two sections provide a detailed background organized in several subsections. Section 2 surveys early attempts at bridging possibility theory and modal logics. Section 3 offers overviews of MEL and its extensions with objective formulas. Section 4 gives a comparative discussion of MEL and $\mathrm{MEL}^{+}$vs. epistemic and rough set logics and proposes a square of opposition-based view of modal logic, possibility theory, and rough sets whose logic obey the axioms of modal system S5. Section 5 is dedicated to the joint extension of $\mathrm{MEL}^{+}$and GPL to $\mathrm{GPL}^{+}$, as well as to the joint extension of $\mathrm{MEL}^{++}$and $\mathrm{GPL}^{+}$to $\mathrm{GPL}^{++}$; soundness and completeness results are established.

\section{Background}

This background section is organized into two pieces. First, we summarize basics of possibility theory (Dubois \& Prade, 1988a, 2014). Then different early attempts at bridging possibility theory and modal logic are reviewed. In the whole paper, we consider a standard propositional language $\mathcal{L}$ with formulas $\varphi, \psi, \ldots$, built up from a finite set of propositional variables $\mathcal{V}=$ $\left\{p_{1}, \ldots, p_{k}\right\}$ along with the Boolean connectives of conjunction and negation $\neg$. As usual, a disjunction $\varphi \vee \psi$ stands for $\neg(\neg \varphi \wedge \neg \psi)$ and an implication $\varphi \rightarrow \psi$ stands for $\neg \varphi \vee \psi$. Further we use $T$ to denote $\varphi \vee \neg \varphi$ for some fixed formula $\varphi$, and $\perp$ to denote $\neg \top$. Consider a set of possible worlds $\Omega$, here, interpretations of the propositional language $\mathcal{L}$. $\Omega$ consists of the set of mappings $w: \mathcal{L} \rightarrow\{0,1\}$ conforming to the rules of classical propositional 
logic. We denote by $w \models \varphi$ when $w(\varphi)=1$ and say that $w$ is a model of $\varphi$.

\subsection{Possibility theory}

A possibility distribution is a mapping $\pi: \Omega \rightarrow U$ that assigns to each possible world $w$ a value $\pi(w)$ from a totally ordered uncertainty scale $\langle U, \leq$ $, 0,1\rangle$. We will furthermore assume $U$ to be such that $\{0,1\} \subseteq U \subseteq[0,1]$ and closed by the negation function $n(x)=1-x$. Such a mapping $\pi$ represents a plausibility ranking of possible worlds according to an agent, and thus it represents an epistemic state, adopting the following conventions:

- $\pi(w)=1$ if $w$ is fully plausible;

- $\pi(w)=0$ if $w$ is rejected as a possible world;

- $\pi(w) \leq \pi\left(w^{\prime}\right)$ if $w^{\prime}$ is at least as plausible as $w$.

A consistent agent is such that $\pi(\omega)=1$ for some $\omega \in \Omega$, in such a case we say that $\pi$ is called a normalised possibility distribution. In the following, we will assume possibility distributions to be normalised. If the range of $\pi$ is $\{0,1\}$, then $\pi$ is the characteristic function of some epistemic state, a non-empty subset $E$ of $\Omega$.

A possibility distribution $\pi: \Omega \rightarrow U$ induces a pair of dual possibility and necessity measures on propositions, defined respectively as:

$$
\begin{gathered}
\Pi(\varphi):=\sup \{\pi(w) \mid w \in \Omega, w \models \varphi\} \\
N(\varphi):=\inf \{1-\pi(w) \mid w \in \Omega, w \not \models \varphi\} .
\end{gathered}
$$

They are dual in the sense that $\Pi(\varphi)=1-N(\neg \varphi)$ for every proposition $\varphi$. The degree $\Pi(\varphi)$ expresses the extent to which there is no impediment to having $\varphi$ true. The case when $\Pi(\varphi)=\Pi(\neg \varphi)=1$ expresses total ignorance about proposition $\varphi$. In contrast, $N(\varphi)$ is the degree of certainty that $\varphi$ is true. Characteristic axioms are $\Pi(\varphi \vee \psi)=\max (\Pi(\varphi), \Pi(\psi))$ for possibility measures and $N(\varphi \wedge \psi)=\min (N(\varphi), N(\psi))$ for necessity measures. Clearly,

- $N(\varphi)>0$ implies $\Pi(\varphi)=1$ and $N(\neg \varphi)=0$.

- $N(\varphi)>0$ if and only if $\varphi$ is true in all the most plausible worlds according to $\pi$.

- $N(\varphi)=1$ if and only if $\varphi$ is true in all the non-completely impossible worlds according to $\pi$. 
- The set of formulas $\varphi$ such that $N(\varphi) \geq \theta$ for any threshold $\theta \neq 0$ is deductively closed.

So, necessity measures are good candidates to model the idea of accepted belief.

\subsection{Early attempts at bridging possibility theory and modal logics}

The first attempt at bridging possibility theory with modal logic can be found in a paper co-authored by L. Fariñas del Cerro (Fariñas del Cerro \& H. Prade, 1986). This paper establishes a formal parallel between rough sets and twofold fuzzy sets (Dubois \& Prade, 1987). The latter model illknown sets of objects having fuzzy attribute values, in terms of possibility and necessity of membership. Then, taking advantage of the existence of the modal logic DAL for rough sets (Fariñas del Cerro \& Orłowska, 1985) and of a modal logic view of incomplete information databases (Lipski, 1981), the paper discusses some possible options for a modal logic agreeing with possibility theory and with the issue of dealing with incomplete information (for twofold fuzzy sets) rather than indiscernibility due to the lack of attributes (as in the case of rough sets).

A couple of years later, the idea of building a modal logic from a graded accessibility relation between different incomplete states of knowledge was investigated in detail in the case of binary-valued possibility theory and suggested for the graded case (Dubois et al., 1988). Then a state of knowledge $s_{2}$ is accessible from a state $s_{1}$ if and only if the information in state $s_{1}$ is consistent with the information in state $s_{2}$, but more incomplete (which was formalized as a set inclusion in the binary-valued case). In the general case, the inclusion becomes a matter of degree and the accessibility relation becomes graded. But the underlying axiom system remained an open issue.

Another attempt at the semantical level at bridging uncertainty theories with modal logic can be found in (Resconi et al., 1992; Resconi et al, 1996; Resconi et al., 1993); it includes the cases of possibility theory (Harmanec \& Klir, 1994b) and Shafer theory of evidence (Harmanec \& Klir, 1994a). In the case of possibility theory, the authors use an accessibility relation assumed to be transitive and complete (connected), which corresponds to modal system S43. Necessity and possibility are built as ratios of the number of worlds in which the corresponding propositions are true.

Note that as early as in 1973 (5 years before Zadeh's possibility theory appeared), Lewis (1973a) introduced comparative possibility relations 
$\succeq$ whereby $\varphi \succeq \psi$ is supposed to mean that $\varphi$ is at least as possible as $\psi$. These relations are total preorders such that $T \succ \perp$ (where $\succ$ is the strict part of $\succeq$ ), $\varphi \succeq \perp$, and the following property holds: $\varphi \succeq \psi$ implies $\varphi \vee \chi \succeq \psi \vee \chi$. Noticeably the only set-functions representing these relations are possibility measures (Dubois, 1986). Lewis (1973b) introduced full-fledged modal logics for encoding comparative statements of the form $\varphi \succeq \psi$ (and their Boolean combinations). One of these logics turned out to exactly capture possibility theory.

However, but for Lewis early construction, whose relevance to possibility theory is clear, there was no direct bridge between plain modal logic and Zadeh's possibility theory, whereby modal formulas could be evaluated by possibility distributions representing epistemic states, until recently.

\section{The logic MEL and its KD45-like extensions}

This section completes the background by providing a brief introduction to the simple logic of incomplete knowledge MEL, and to its extensions $\mathrm{MEL}^{+}$ and $\mathrm{MEL}^{++}$, comparing them to epistemic and rough set logics.

\subsection{MEL: a simple epistemic logic}

The usual truth values true (1) and false (0) assigned to propositions are of ontological nature (which means that they are part of the definition of what we call proposition), whereas assigning to a proposition a value whose meaning is expressed by the word unknown sounds like having an epistemic nature: it reveals a knowledge state according to which the truth value of a proposition (in the usual Boolean sense) in a given situation is out of reach (for instance one cannot compute it, either by lack of computing power, or due to a sheer lack of information). It corresponds to an epistemic state for an agent that can neither assert the truth of a Boolean proposition nor its falsity.

Admitting that the concept of "unknown" refers to a knowledge state rather than to an ontic truth value, we may start with Boolean logic where asserted formulas are interpreted as beliefs, and add to its syntax the capability of stating that we ignore the truth value ( 1 or 0$)$ of propositions. The natural framework to syntactically encode statements about knowledge states of classical propositional logic (CPL) statements is modal logic, and in particular, the logic KD. Nevertheless, if one only wants to reason about, e.g., the beliefs of another agent, a very limited fragment of this language 
is needed. The logic MEL (Banerjee \& Dubois, 2009, 2014) was defined for that purpose.

Given a standard propositional language $\mathcal{L}$, consider another propositional language $\mathcal{L}_{\square}$ whose set of propositional variables is of the form $\mathcal{V}_{\square}=\{\square \varphi \mid \varphi \in \mathcal{L}\}$ to which the classical connectives can be applied. It is endowed with a modality operator expressing certainty, that encapsulates formulas in $\mathcal{L}$. As usual, $\diamond \varphi$ is short for $\neg \square \neg \varphi$. Formulas in $\mathcal{L}_{\square}$ are clearly modal formulas of depth 1 , denoted by $\Phi, \Psi, \ldots$.

MEL is a propositional logic on the language $\mathcal{L}_{\square}$ with the following semantics. Let $\Omega$ be the set of classical interpretations for the propositional language $\mathcal{L}$. A model of a propositional formula $\varphi \in \mathcal{L}$ is an element of $\Omega$, we will denote by $\operatorname{Mod}(\varphi) \subseteq \Omega$ the set of models of $\varphi$. In contrast, models (or interpretations) for MEL correspond to epistemic states, which are simply subsets $\emptyset \neq E \subseteq \Omega$. The truth-evaluation rules of formulas of $\mathcal{L}_{\square}$ in a given epistemic model $E$ are defined as follows:

- $E \models \square \varphi$ if $E \subseteq \operatorname{Mod}(\varphi)$

- $E \models \neg \Phi \quad$ if $\quad E \not \models \Phi$

- $E \models \Phi \wedge \Psi \quad$ if $\quad E \models \Phi$ and $E \models \Psi$

The intuition is that if the epistemic state of an agent is $E$ and $E \subseteq \operatorname{Mod}(\varphi)$, then this agent believes that $\varphi$ is true. Note that contrary to what is usual in modal logic, modal formulas are not evaluated on particular interpretations of the language $\mathcal{L}$ because modal formulas in MEL do not refer to the actual world.

The notion of logical consequence is defined as usual: Let $\Gamma$ be a set of $\mathcal{L}_{\square}$-formulas and $\Phi$ be another such formula; then $\Gamma \models \Phi$ if, for every epistemic model $E, E \models \Phi$ whenever $E \models \Psi$ for all $\Psi \in \Gamma$.

MEL can be axiomatized in a rather simple way (see (Banerjee \& Dubois, 2014)). The following is a possible set of axioms for MEL in the language of $\mathcal{L}_{\square}$ :

(CPL) Axioms of CPL for $\mathcal{L}_{\square}$-formulas

$(\mathrm{K}) \square(\varphi \rightarrow \psi) \rightarrow(\square \varphi \rightarrow \square \psi)$

(D) $\square \varphi \rightarrow \diamond \varphi$

(Nec) $\square \varphi$, for each $\varphi \in \mathcal{L}$ that is a CPL tautology, i.e., if $\operatorname{Mod}(\varphi)=\Omega$. 
The only inference rule is modus ponens. The corresponding notion of proof, denoted by $\vdash$, is defined as usual from the above set of axioms and modus ponens.

This set of axioms provides a sound and complete axiomatization of MEL, that is, it holds that, for any set of MEL formulas $\Gamma \cup\{\Phi\}, \Gamma \models \Phi$ iff $\Gamma \vdash \Phi$. This is not surprising: MEL is just a standard propositional logic with additional axioms, whose propositional variables are the formulas of another propositional logic, and whose interpretations are subsets of interpretations of the latter.

Note that the statement $\square \varphi$ in MEL can be expressed as $\varphi \succ \perp$ in (Lewis, 1973b) systems. See for instance (Touazi et al., 2015) for a more precise discussion. Besides, the semantics of MEL not only equates the statement $\square \varphi$ with $N(\varphi)=1$ in the sense of a necessity measure, but also with the statement $P(\varphi)=1$ in the sense of a probability measure, and $\operatorname{Bel}(\varphi)=1$ in the sense of a Shafer's belief function (Banerjee \& Dubois, 2014).

The language MEL was originally supposed to encode the following situation (Banerjee \& Dubois, 2009). There are two agents, one of which, say $\mathcal{A}$, reasons about some beliefs possessed by another agent $\mathcal{B}$, the former is aware of, on the basis of the testimony of the latter. Namely, $\mathcal{A}$ partially knows what the other agent believes. A belief base in MEL typically contains the testimony of agent $\mathcal{B}$, namely propositions agent $\mathcal{B}$ has declared to believe $(\square \alpha)$, some that he explicitly does not know $(\diamond \alpha \wedge \diamond \neg \alpha)$, and finally some other propositions that agent $\mathcal{A}$ is aware the agent $\mathcal{B}$ knows the truth-value of, without guessing which one $(\square \alpha \vee \square \neg \alpha)$. The logic MEL enables agent $\mathcal{A}$ to infer more beliefs agent $\mathcal{B}$ possesses but did not reveal. Such a (meta)belief base for agent $\mathcal{A}$ about $\mathcal{B}$ corresponds to a meta-epistemic state of $\mathcal{A}$ modelled by a set of possible epistemic states for agent $\mathcal{B}$. Namely $\mathcal{A}$ knows that the epistemic state of $\mathcal{B}$ is one of them without knowing which one.

\subsection{From MEL to MEL $^{+}$}

MEL has been extended in (Banerjee et al., 2014) to allow dealing with not only subjective formulas that express an agent's beliefs, but also objective formulas (i.e., non-modal formulas) that express propositions that hold true in the actual world (whatever it might be). The extended language will be denoted by $\mathcal{L}_{\square}^{+}$, and it thus contains both propositional and modal formulas. It exactly corresponds to the non-nested fragment of the language of usual modal logic.

More precisely, the language $\mathcal{L}_{\square}^{+}$of $\mathrm{MEL}^{+}$extends $\mathcal{L}_{\square}$ and is defined by 
the following formation rules:

- If $\varphi \in \mathcal{L}$ then $\varphi, \square \varphi \in \mathcal{L}_{\square}^{+}$

- If $\Phi, \Psi \in \mathcal{L}_{\square}^{+}$then $\neg \Phi, \Phi \wedge \Psi \in \mathcal{L}_{\square}^{+}$

$\diamond \varphi$ is again defined as an abbreviation of $\neg \square \neg \varphi$. Note that $\mathcal{L} \subseteq \mathcal{L}_{\square}^{+}$(while $\mathcal{L} \cap \mathcal{L}_{\square}=\emptyset$ ) and that in $\mathcal{L}_{\square}^{+}$there are no formulas with nested modalities.

Semantics for $\mathrm{MEL}^{+}$are now given by "pointed" MEL epistemic models, i.e., by structures $(w, E)$, where $w \in \Omega$ and $\emptyset \neq E \subseteq \Omega$. The truth-evaluation rules of formulas of $\mathcal{L}_{\square}^{+}$in a given structure $(w, E)$ are defined as follows:

- $(w, E) \models \varphi$ if $w \in \operatorname{Mod}(\varphi)$, in case $\varphi \in \mathcal{L}$

- $(w, E) \models \square \varphi$ if $E \subseteq \operatorname{Mod}(\varphi)$

- usual rules for $\neg$ and $\wedge$

Logical consequence is defined as usual: $\Gamma \models \Phi$ if, for every pointed model $(w, E)$, it holds that $(w, E) \models \Phi$ whenever $(w, E) \models \Psi$ for all $\Psi \in \Gamma$. The axioms for $\mathrm{MEL}^{+}$in the language of $\mathcal{L}_{\square}^{+}$are the same as for MEL (CPL, $\mathrm{K}, \mathrm{D}, \mathrm{Nec}$ ), except that the language of $\mathrm{MEL}^{+}$strictly includes the one of MEL and $\mathcal{L}$. The only inference rule is modus ponens. ${ }^{2}$

It can be proven that the above axiomatization of $\mathrm{MEL}^{+}$is sound and complete with respect to the intended semantics, as defined above. Moreover, as it could be expected, if we call $\mathrm{MEL}^{++}$the extension of $\mathrm{MEL}^{+}$with the axiom:

$$
\text { (T) } \square \varphi \rightarrow \varphi
$$

then it can be shown that $\mathrm{MEL}^{++}$is sound and complete with respect to the class of reflexive pointed epistemic models $(w, E)$, i.e., where $w \in E$. Note that as axiom $\mathrm{T}$ cannot be expressed in MEL, the latter logic can be indifferently intepreted as a doxastic or an epistemic logic, as we cannot tell belief from knowledge in this setting, due to lack of expressiveness.

Actually, MEL, $\mathrm{MEL}^{+}$and $\mathrm{MEL}^{++}$capture different non-nested fragments of the normal modal logics of belief KD, KD4, KD45 and S5 (see e.g., (Chellas, 1980) for details). Indeed, in (Banerjee et al., 2014) the following relationships are shown:

\footnotetext{
${ }^{2}$ An equivalent presentation could be to replace (Nec) by the usual Necessitation rule in modal logics, but restricted to tautologies of propositional logic: if $\varphi \in \mathcal{L}$ is a theorem, derive $\square \varphi$.
} 
- Let $\varphi$ be a formula from $\mathcal{L}_{\square}$. Then MEL $\vdash \varphi$ iff $L \vdash \varphi$, for $L \in\{\mathrm{KD}, \mathrm{KD} 4, \mathrm{KD} 45, \mathrm{~S} 5\}$.

- Let $\varphi$ be a formula from $\mathcal{L}_{\square}^{+}$. Then $\mathrm{MEL}^{+} \vdash \varphi$ iff $L \vdash \varphi$, for $L \in\{\mathrm{KD}, \mathrm{KD} 4, \mathrm{KD} 45\}$.

- Let $\varphi$ be a formula from $\mathcal{L}_{\square}^{+}$. Then, $\mathrm{MEL}^{++} \vdash \varphi$ iff $\mathrm{S} 5 \vdash \varphi$.

Moreover, by recalling the well-known result that any formula of KD45 and S5 is logically equivalent to another formula without nested modalities, the following stronger relationships hold:

- For any arbitrary modal formula $\varphi$, there is a formula $\varphi^{\prime} \in \mathcal{L}_{\square}^{+}$such that $\mathrm{KD} 45 \vdash \varphi$ iff $\mathrm{MEL}^{+} \vdash \varphi^{\prime}$.

- For any arbitrary modal formula $\varphi$, there is a formula $\varphi^{\prime} \in \mathcal{L}_{\square}^{+}$such that $\mathrm{S} 5 \vdash \varphi$ iff $\mathrm{MEL}^{++} \vdash \varphi^{\prime}$.

Remark 1 In Petruszczak (2009) it is indicated that simplified Kripke frames could indeed be used for the semantics of systems K45, KB5 and KD 45, using subsets of propositional valuations in place of relations, as we proposed. He proves it by constructing specific accessibility relations equivalent to such subsets, as in (Banerjee 8 Dubois, 2009) for MEL, while the completeness proof in (Banerjee 85 Dubois, 2014) (and here) is direct.

Let us discuss the possible use of these extensions of MEL for agentbased reasoning. In $\mathrm{MEL}^{+}$, agent $\mathcal{A}$ is allowed to add what is known about the real world in the form of standard propositions. So $\alpha \wedge \square \neg \alpha$ means that agent $\mathcal{A}$ considers $\alpha$ is true, while he knows that agent $\mathcal{B}$ believes it is false. Under this set-up, a $\mathrm{MEL}^{+}$model $(w, E)$ is interpreted as the fact that $\mathcal{A}$ envisages the real world to be $w$ and the epistemic state of $\mathcal{B}$ to be $E$. If $\mathcal{A}$ considers that $\mathcal{B}$ 's beliefs are always correct, the former can assume axiom $\mathrm{T}$ is valid, thus he reasons in $\mathrm{MEL}^{++}$to strengthen his own knowledge of the real world. Alternatively, $\mathcal{A}$ may mistrust $\mathcal{B}$ and may wish to take advantage of knowing wrong beliefs of $\mathcal{B}$.

\section{MEL and $\mathrm{MEL}^{+}$vs. other logics of incomplete information}

The logic S5 has been extensively used as the archetype of epistemic logic, and also the natural logical framework for rough sets. In both cases, the 
semantics is described in terms of equivalence relations, whose properties are captured by $\mathrm{S} 5$ axioms. In this section we compare $\mathrm{MEL}^{+}$to such logics. Finally we indicate how the square of opposition captures and exhibits the roots of the formal similarities underlying modal logic, possibility theory, and rough sets.

\subsection{Epistemic logic and accessibility relations: a critique}

In contrast with MEL and $\mathrm{MEL}^{+}$, the usual semantics of S5 (Halpern et al., 2003) considers the epistemic state of an agent as being modelled by an equivalence relation $R$ on a set of possible worlds $\Omega .^{3}$ The statement $w R w^{\prime}$ reads "world $w^{\prime}$ is accessible from $w$ ". The world $w^{\prime}$ is said to be an epistemic or doxastic alternative to world $w$ for the agent, depending on whether knowledge or belief is the considered attitude. There are various attempts to make sense of this relation, such as: the agent cannot distinguish $w$ from $w^{\prime}$; or $w^{\prime}$ is a possible state of affairs from the point of view of what the agent knows in $w$, etc. The underlying idea seems to be that "the set of worlds accessible to ${ }^{4}$ by an agent depends on his or her informational resources at that instant" (Hendricks \& Symons, 2015).

However this view, which seems to be shared by many scholars, is not so easy to grasp. Interpreting "accessible worlds" as worlds compatible with the agent epistemic state, we can assume that the epistemic state of the agent depends on his or her informational resources at that instant (in MEL this possibility is not considered). But it is not clear that the agent is aware of his own informational resources to the point of articulating them in the same language as the one he uses to speak about the current states of affairs. If $w$ stands for an objective state of facts, it may not include the particulars of the agent. The epistemic state of the agent depends on many hidden internal features of the agent but his current observations about the actual world $w$ refer to something external, the agent is focused on. It is not clear that the vocabulary used to describe the actual world $w$ is rich enough to also account for the inner state (of health or informational resources) of the agent that holds some beliefs about $w$. For instance suppose the agent has incomplete information on the outcome of a coin flipping round after the toss: this epistemic state will generally not depend on the outcome of the toss (like "if the result is heads then he knows it, otherwise he does not

\footnotetext{
${ }^{3}$ See (Fagin \& Vardi, 1985) for an alternative semantics that makes the internal structure of possible worlds more explicit, encoding both objective facts and agents' mental states in a possible world.

${ }^{4}$ i.e., considered possible.
} 
know").

In a nutshell, while an equivalence class of $R$ represents contextdependent knowledge of the agent, it is not clear that this contextual dependence is part of the agent's knowledge about himself, let alone about another agent. So in the epistemic logic approach the accessibility relation seems to be a circular notion, where possible worlds seem to include the description of the agent mental circumstances as well as the description of his epistemic state regarding the problem he considers. This view may make sense when introspection is the main issue (the agent being partially unaware of his own knowledge), but it seems to be at odds with the problem of an agent reflecting about other agents' knowledge, as in the set-up for MEL and $\mathrm{MEL}^{+}$. As the set-up for MEL is not introspective, this relational semantics looks like a questionable artifact for this logic, where we assume agents are aware of their own knowledge and lack of knowledge. Note that if $R=\Omega \times \Omega$ one can only distinguish between tautologies (i.e., $\square \varphi$ where $\varphi$ is a CPL tautology), contradictions and contingent modal propositions.

One may extend the MEL set-up by considering a separate set of possible mental dispositions $S$ corresponding to "informational resources" (due to specific situations or circumstances) an agent can access at a particular moment. On the other hand, $\Omega$ encodes the question the agent is concerned with at that moment; it pertains to the outside world, so $S \cap \Omega=\emptyset$. The accessibility relation $R$ is relating $S$ to $\Omega$, namely $E=R(s) \subseteq \Omega$ is the epistemic state of the agent when his mental disposition is $s$. Note that, under this view, there is no point of $R$ being an equivalence relation. But this extension assumes that the set $S$ of mental dispositions of the agent is known and observable by another agent.

\subsection{Comparison to logics of rough sets}

The semantics of S5 in terms of equivalence relations (Chellas, 1980) makes it in fact the natural logical setting for rough sets (Orłowska, 1985). Pawlak (1991)'s rough sets (see also (Pawlak \& Skowron, 2007)) are based on the notion of approximation spaces $(\Omega, R)$, where $R$, called the indiscernibility relation, is an equivalence relation on the domain $\Omega$ of discourse. The idea is that due to the lack of complete information about the objects in the domain, it is likely that many of the objects are indistinguishable from each other. This is patent in information systems $I:=\left(\Omega, A t, V_{A t}, f\right)$, where $A t$ is a set of attributes, $V_{A t}$ a set of values for the attributes in $A t$, and $f: \Omega \times A t \rightarrow V_{A t}$ a function assigning values for attributes to objects of the domain. $I$ then induces an indiscernibility relation $R$ corresponding to 
every subset $B \subseteq A t$ :

$$
(x, y) \in R \text { if and only if } f(x, b)=f(y, b) \text {, for all } b \in B .
$$

The lower and upper approximations of a subset $X$ of the domain with respect to $R$ are defined as

$$
\underline{X}:=\{x \in \Omega: R(x) \subseteq X\} ; \bar{X}:=\{x \in \Omega: R(x) \cap X \neq \emptyset\} .
$$

Sets with identical approximations are said to be roughly equal, and for any $X$, the collection of all subsets of the domain roughly equal to $X$ is termed a rough set by Pawlak (1991).

Any logic of rough sets thus has an essential modal nature, the necessity and possibility operators capturing the lower and upper approximations in the rough set semantics respectively. In fact, for any S5 Kripke model $M=$ $(\Omega, w, R)$, one may observe that $\operatorname{Mod}(\square \varphi)=\operatorname{Mod}(\varphi)$ and $\operatorname{Mod}(\diamond \varphi)=$ $\overline{\operatorname{Mod}(\varphi)}$, where $\operatorname{Mod}(\varphi):=\{w \in \Omega: w \models \varphi\}$. However, unlike MEL or $\mathrm{MEL}^{+}$, rough set logics make use of the full modal language, that is, nested modalities are allowed. For instance one would use a formula such as $\diamond(p \wedge \square q)$ to refer to a set $\overline{(X \cap \underline{Y})}$.

This approach can easily be extended to rough set models based on a relation that is not necessarily an equivalence one (Ciucci \& Dubois, 2014; Yao \& Lin, 1996; Yao et al., 1997). These logics remain modal, and use nested modalities. Indeed, it is well known in modal logic (Chellas, 1980) that, once the basic axioms (PL) and $(\mathrm{K})$ are fixed, each additional modal axiom corresponds to a different property of the accessibility relation.

\subsection{Possibility theory, rough sets and modal logics: a square of opposition viewpoint}

Recent studies (Dubois et al., 2015) have pointed out that many artificial intelligence knowledge representation settings are sharing the same structures of opposition that extend or generalize the traditional square of opposition which dates back to Aristotle, and whose logical interest has been rediscovered more than one decade ago (Béziau, 2003). The traditional square, pictured in Fig. 1, is usually denoted by the letters $\mathbf{A}, \mathbf{I}$ (affirmative half) and $\mathbf{E}, \mathbf{O}$ (negative half). The names of the vertices come from a traditional Latin reading: AffIrmo, $\mathrm{n} \mathbf{E g} \mathbf{O}$ ). This square involves four logically related statements exhibiting universal or existential quantifications: a statement $\mathbf{A}$ of the form "every $x$ is $p$ " is negated by the statement $\mathbf{O}$ "some $x$ is not $p$ ", while a statement like $\mathbf{E}$ "no $x$ is $p$ " is clearly in even stronger opposition to 
the first statement $(\mathbf{A})$. These three statements, together with the negation of the last one, namely $\mathbf{I}$ "some $x$ is $p$ ", give birth to the Aristotelian square of opposition in terms of quantifiers $\mathbf{A}: \forall x p(x), \mathbf{E}: \forall x \neg p(x), \mathbf{I}: \exists x p(x)$, $\mathbf{O}: \exists x \neg p(x)$.

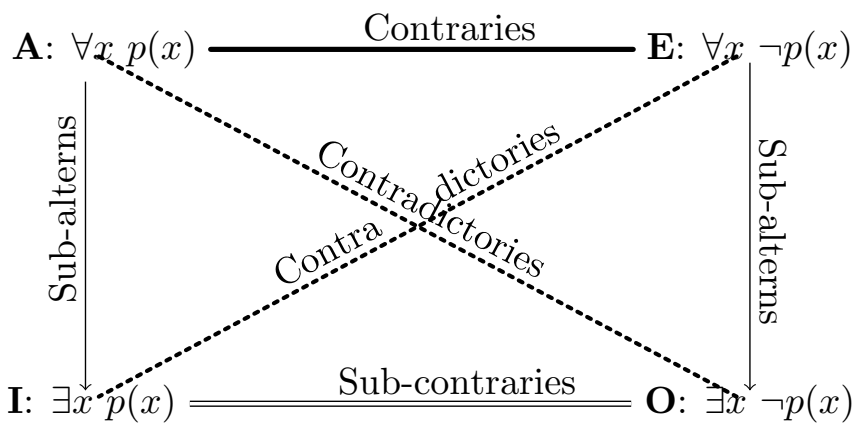

Figure 1: Square of opposition

Note that we assume that some $x$ do exist, thus avoiding existential import problems in Fig. 1. The different edges and diagonals of the square exhibit simple logical relations:

i) $\mathbf{A}$ and $\mathbf{O}$, as well as $\mathbf{E}$ and $\mathbf{I}$, are the negation of each other;

ii) $\mathbf{A}$ entails $\mathbf{I}$, and $\mathbf{E}$ entails $\mathbf{O}$;

iii) $\mathbf{A}$ and $\mathbf{E}$ cannot be true together,

iv) $\mathbf{I}$ and $\mathbf{O}$ cannot be false together.

Another well-known instance of this square is in terms of the necessary $(\square)$ and possible $(\diamond)$ modalities, with the following reading $\mathbf{A}: \square p, \mathbf{E}$ : $\square \neg p, \mathbf{I}: \diamond p, \mathbf{O}: \diamond \neg p$, where $\diamond p==_{d e f} \neg \square \neg p$ (with $p \neq \perp, \top$ ). Then the entailment from $\mathbf{A}$ to $\mathbf{I}$ is nothing but the axiom (D) in modal logic, namely $\square p \rightarrow \diamond p$. This reading has an easy counterpart in terms of binaryvalued possibility theory replacing $\square p$ by $N(\operatorname{Mod}(p))$ and $\diamond p$ by $\Pi(\operatorname{Mod}(p))$ where $\operatorname{Mod}(p)$ is the set of models of proposition $p$ (Dubois \& Prade, 2012). This framework can be extended to graded possibility theory using a graded extension of the square of opposition (Ciucci et al., 2015a).

A relation-based reading of the square of opposition has been proposed in (Ciucci et al., 2014, 2015a). Let us now consider a binary relation $R$ on a Cartesian product $X \times Y$ (one may have $Y=X$ ). We assume $R \neq \emptyset$. Let $x R$ denote the set $\{y \in Y \mid(x, y) \in R\}$, and $R y=\{x \in X \mid(x, y) \in R\}$. We 
write $x R y$ when $(x, y) \in R$ holds, and $\neg(x R y)$ when $(x, y) \notin R$. Moreover, we assume that $\forall x, x R \neq \emptyset$, which means that the relation $R$ is serial, namely $\forall x, \exists y$ such that $x R y$. We further assume that the complementary relation $\bar{R}(x \bar{R} y$ iff $\neg(x R y))$, and its transpose are also serial, i.e., $\forall x, x R \neq$ $Y$ and $\forall y, R y \neq X$. These conditions enforce a non trivial relation between $X$ and $Y$. In the following, set complementations are denoted by means of overbars.

Let $S$ be a subset of $Y$. We assume $S \neq \emptyset$ and $S \neq Y$. The relation $R$ and the subset $S$, also considering its complement $\bar{S}$, give birth to the two following subsets of $X$, namely the (left) images of $S$ and $\bar{S}$ by $R$

$$
\begin{aligned}
& R(S)=\{x \in X \mid \exists s \in S, x R s\}=\{x \in X \mid S \cap x R \neq \emptyset\}=\bigcup_{s \in S} R s \\
& R(\bar{S})=\{x \in X \mid \exists s \in \bar{S}, x R s\}=\bigcup_{s \in \bar{S}} R s
\end{aligned}
$$

and their complements

$$
\begin{aligned}
& \overline{R(S)}=\{x \in X \mid \forall s \in S, \neg(x R s)\}=\overline{\bigcup_{s \in S} R s}=\bigcap_{s \in S} \overline{R s}=\bigcap_{s \in S} \bar{R} s \\
& \overline{R(\bar{S})}=\{x \in X \mid \forall s \in \bar{S}, \neg(x R s)\}=\{x \in X \mid x R \subseteq S\}=\overline{\bigcup_{s \in \bar{S}} R s}=\bigcap_{s \in \bar{S}} \bar{R} s
\end{aligned}
$$

The four subsets thus defined can be nicely organized into a square of opposition, see Fig. 2. Indeed, it can be checked that the set counterparts of the logical relations existing between the logical statements of the traditional square of opposition still hold here.

Namely,

- $\overline{R(\bar{S})}$ and $R(\bar{S})$ are complements of each other, as are $\overline{R(S)}$ and $R(S)$; they correspond to the diagonals of the square;

- $\overline{R(\bar{S})} \subseteq R(S)$, and $\overline{R(S)} \subseteq R(\bar{S})$, thanks to condition $\forall x, x R \neq \emptyset$. These inclusions are represented by vertical arrows in Fig. 2;

- $\overline{R(\bar{S})} \cap \overline{R(S)}=\emptyset$; this empty intersection corresponds to the thick line in Fig. 2, and one may have $\overline{R(\bar{S})} \cup \overline{R(S)} \neq X$;

- $R(S) \cup R(\bar{S})=X$; this full union corresponds to the double thin line in Fig. 2, and one may have $R(S) \cap R(\bar{S}) \neq \emptyset$. 


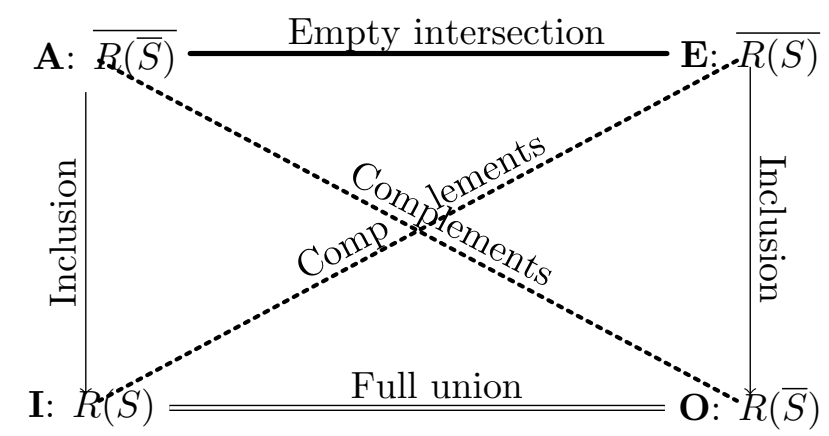

Figure 2: Square of opposition induced by a relation $R$ and a subset $S$

Conditions (iii)-(iv) hold also thanks to the serial property of $R$.

Note that this fits with a modal logic reading of this square where $R$ is viewed as an accessibility relation defined on $X \times X$, and $S$ as the set of models of a proposition $p$. Indeed, $\square p$ (resp. $\diamond p$ ) is true in world $x$ means that $p$ is true at every (resp. at some) possible world accessible from $x$; this corresponds to $\overline{R(\bar{S})}$ (resp. $R(S)$ ) which is the set of worlds where $\square p$ (resp. $\diamond p$ ) is true. Moreover, the entailment from $\mathbf{A}$ to $\mathbf{I}$ is the axiom (D) of modal logic which is known to require serial accessibility relations (Chellas, 1980).

Note that the relation $R$ is serial if and only if $\overline{R(\bar{S})} \subseteq R(S)$. An interesting instantiation is in terms of rough sets (Ciucci et al., 2014), where in the classical case $R$ is an equivalence relation. Then given the above definitions, we recognize that

- $R(S)$ is the upper approximation of $S$ wrt the relation $R$;

- $\overline{R(\bar{S})}$ is the lower approximation of $S$ wrt the relation $R$;

- $\overline{R(S)}$ is the exterior region of $S$;

- $R(\bar{S})$ is the complement of the lower approximation of $S$.

The above notions can be extended to fuzzy rough sets (Dubois \& Prade, 1988b), if we replace the approximation operators by fuzzy ones. We obtain fuzzy extensions of box and diamond operators on crisp or fuzzy sets, also studied by Thiele (1998). A study of fuzzy rough sets in relation to the square of opposition appears in (Ciucci et al., 2015b). 


\section{$5 \quad \mathrm{GPL}^{+}$: extending generalized possibilistic logic with objective formulas}

A natural generalization of MEL is to extend epistemic states $E \subseteq \Omega$ to rankings of possible worlds in terms of plausibility. This can be done by means of possibility distributions. Possibilistic logic (see e.g. (Dubois et al., 1994; Dubois \& Prade, 2004, 2014)), nowadays a well-known uncertainty logic, was initially devised to reason with graded beliefs on classical propositions by means of necessity and possibility measures. For instance, the necessity fragment of possibilistic logic deals with weighted formulas $(\varphi, r)$, where $\varphi$ is a classical proposition and $r \in U$ is a weight, interpreted as a lower bound for the necessity degree of $\varphi$. It has a very simple axiomatization:

$(\mathrm{CPL})(\varphi, 1)$, for $\varphi$ being a tautology of $\mathrm{CPL}$

$(\operatorname{GMP})$ from $(\varphi, r)$ and $(\varphi \rightarrow \psi, s)$ derive $(\psi, \min (r, s))$

(W) from $(\varphi, r)$ derive $(\varphi, s)$, if $s \leq r$

where GMP stands for generalised modus ponens, and (W) is a weakening rule.

The aim of this section is to present the graded extension of MEL called GPL, an extension of possibilistic logic which uses graded modalities, and to introduce the logic $\mathrm{GPL}^{+}$which similarly extends $\mathrm{MEL}^{+}$in an S5-like language.

\subsection{Generalized possibilistic logic}

A graded extension of MEL capturing possibilistic logic has been proposed under the name Generalized Possibilistic Logic, GPL for short, in (Dubois et al., 2012). To deal with graded possibility and necessity they fix a finite scale of uncertainty values $\Lambda=\left\{0, \frac{1}{k}, \frac{2}{k}, \ldots, 1\right\}$, where $k \in \mathbb{N} \backslash\{0\}$. Moreover we let $\Lambda^{+}=\Lambda \backslash\{0\}$, and if $a \in \Lambda^{+}$, we denote by $p(a)$ the value in the scale that precedes $a$, i.e. $p\left(\frac{i}{k}\right)=\frac{i-1}{k}$.

Then, based on a propositional language $\mathcal{L}$, we define another language $\mathcal{L}_{\square}^{k}$ with a pair of modal operators $\square_{a}$ and $\diamond_{a}$ for each value $a \in \Lambda^{+}$, in the style of MEL. More precisely, the language $\mathcal{L}_{\square}^{k}$ of GPL is defined by the following formation rules:

- If $\varphi \in \mathcal{L}$ and $a \in \Lambda^{+}$then $\square_{a} \varphi \in \mathcal{L}_{\square}^{k}$;

- If $\Phi, \Psi \in \mathcal{L}_{\square}^{k}$ then $\neg \Phi, \Phi \wedge \Psi \in \mathcal{L}_{\square}^{k}$. 
In this case, models (epistemic states) are $\Lambda$-valued possibility distributions $\pi: \Omega \rightarrow \Lambda$ on the set $\Omega$ of classical interpretations of the propositional language $\mathcal{L}$. Given such a possibility distribution $\pi$, the satisfaction relation of modal formulas is as follows:

$$
\pi \models \square_{a} \varphi \quad \text { if } \quad N_{\pi}(\varphi)=\min \{1-\pi(w) \mid w \not \models \varphi\} \geq a .
$$

Now since $\Pi(\varphi) \geq a$ is equivalent to $N(\varphi) \leq 1-a$, that is $\neg(N(\varphi) \geq 1-p(a))$, then $\diamond_{a} \varphi$ is defined as an abbreviation of $\neg \square_{b} \neg \varphi$, with $a=1-p(b)$. Note that, like in MEL, $\mathcal{L} \cap \mathcal{L}_{\square}^{k}=\emptyset$ and that in $\mathcal{L}_{\square}^{k}$ there are no formulas with nested modalities.

The semantics of $\diamond_{a} \varphi$ is the natural one, i.e., $\pi \models \diamond_{a} \varphi$ whenever the possibility degree of $\varphi$ induced by $\pi, \Pi(\varphi)=\max \{\pi(w) \mid w \models \varphi\}$, is at least a. A complete axiomatization of GPL is given in (Dubois et al., 2012), an equivalent and shorter axiomatization is given by the following additional set of axioms and rules to those of CPL (Dubois et al., 2014):

$(\mathrm{K}) \square_{a}(\varphi \rightarrow \psi) \rightarrow\left(\square_{a} \varphi \rightarrow \square_{a} \psi\right)$

(D) $\square_{a} \varphi \rightarrow \diamond_{1} \varphi$

(W) $\square_{a_{1}} \varphi \rightarrow \square_{a_{2}} \varphi$, if $a_{1} \geq a_{2}$

(Nec) $\square_{1} \varphi$, for each $\varphi \in \mathcal{L}$ that is a CPL tautology.

The only inference rule is modus ponens.

In this section we extend the language of GPL to allow dealing with not only subjective formulas that express an agent's beliefs, but also objective formulas (i.e., non-modal formulas) expressing propositions that hold true in the actual world (whatever it might be). The extended language will be denoted by $\mathcal{L}_{\square}^{k+}$, and it thus contains both propositional and modal formulas, and combinations thereof. It exactly corresponds to the non-nested fragment of the language of a multi-modal logic.

More precisely, the language $\mathcal{L}_{\square}^{k+}$ of $\mathrm{GPL}^{+}$extends the one of GPL, $\mathcal{L}_{\square}^{+}$, and is defined by the following formation rules:

- If $\varphi \in \mathcal{L}$ and $a \in \Lambda^{+}$then $\varphi, \square_{a} \varphi \in \mathcal{L}_{\square}^{k+}$

- If $\Phi, \Psi \in \mathcal{L}_{\square}^{k+}$ then $\neg \Phi, \Phi \wedge \Psi \in \mathcal{L}_{\square}^{k+}$

$\diamond_{a} \varphi$ is defined as in $\mathcal{L}_{\square}^{k}$. Note that $\mathcal{L} \subseteq \mathcal{L}_{\square}^{k+}$ and that in $\mathcal{L}_{\square}^{k+}$, there are no formulas with nested modalities. 
Semantics for $\mathrm{GPL}^{+}$are given now by "pointed" possibilistic models, i.e., by structures $(w, \pi)$, where $w \in \Omega$ and $\pi: \Omega \rightarrow \Lambda$ such that there is at least one $w \in \Omega$ with $\pi(w)=1$. The truth-evaluation rules of formulas of $\mathcal{L}_{\square}^{k+}$ in a given structure $(w, \pi)$ are defined as follows:

- $(w, \pi) \models \varphi$ if $w \models \varphi$, in case $\varphi \in \mathcal{L}$

- $(w, \pi) \models \square_{a} \varphi$ if $N_{\pi}(\varphi) \geq a$

- usual rules for $\neg$ and $\wedge$

If we let $\pi_{a}=\{w \in \Omega \mid \pi(w) \geq a\}$, note that $(w, \pi) \models \square_{a} \varphi$ whenever $\pi_{1-p(a)} \subseteq \operatorname{Mod}(\varphi)$. Therefore, it becomes clear that each $\square_{a}$ operator is a $\mathrm{MEL}^{+}$modality.

The corresponding logical consequence relation is defined as usual: Let $\Gamma \models \Phi$ if and only if $(w, \pi) \models \Psi$ for all $\Psi \in \Gamma$, then $(w, \pi) \models \Phi$ holds as well, for every structure $(w, \pi)$.

The axioms for $\mathrm{GPL}^{+}$in the language of $\mathcal{L}_{\square}^{k+}$ are the same as those of GPL: Axioms of propositional logic, $\left(\mathrm{K}_{a}\right),\left(\mathrm{D}_{a}\right),(\mathrm{W})$ and $(\mathrm{Nec})$. The only inference rule is modus ponens. We will write $\Gamma \vdash \Phi$ to denote that $\varphi$ can be derived from a set of formulas $\Gamma$ using these axioms and modus ponens. Also, in what follows, we will denote by $\vdash_{C P L}$ the notion of proof of classical propositional language on the language $\mathcal{L}_{\square}^{k+}$ taking all $\square$-formulas as new propositional variables.

To prove completeness, we first recall the following useful lemma that allows to express deductions in $\mathrm{GPL}^{+}$as deductions in CPL. In what follows, $\operatorname{Inst}(A x)$ will denote the set of all instances of axioms $\left(\mathrm{K}_{a}\right),\left(\mathrm{D}_{a}\right),(\mathrm{W})$ and $(\mathrm{Nec})$.

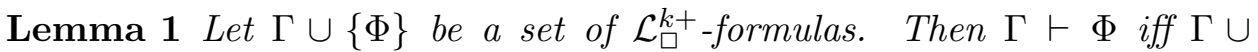
$\operatorname{Inst}(A x) \vdash_{C P L} \Phi$.

Theorem 2 (Soundness and Completeness) For any set of $\mathcal{L}_{\square}^{k+}$ formulas $\Gamma \cup\{\Phi\}$, it holds that $\Gamma \vdash \Phi$ iff $\Gamma \models \Phi$.

Proof: From left to right, it is easy, as usual. For the converse direction, assume $\Gamma \nvdash \Phi$. By the preceding lemma and the completeness of PL, there exists a propositional evaluation $v$ on the whole language $\mathcal{L}_{\square}^{k+}$ (taking $\square$ formulas as genuine propositional variables) such that $v(\Psi)=1$ for all $\Psi \in$ $\Gamma \cup \operatorname{Inst}(A x)$ but $v(\Phi)=0$. We have to build a structure $(w, \pi)$ that it is a model of $\Gamma$ but not of $\Phi$. So, we take $(w, \pi)$ as follows: 
- $w$ is defined as the restriction of $v$ to $\mathcal{L}$, i.e., $w(\varphi)=v(\varphi)$ for all $\varphi \in \mathcal{L}$.

- For each $a \in \Lambda^{+}$, let us first define $E_{1-p(a)}=\bigcap\left\{\operatorname{Mod}(\varphi) \mid v\left(\square_{a} \varphi\right)=\right.$ $1\}$. Then define $\pi: \Omega \rightarrow \Lambda$ as follows: $\pi(w)=\max \left\{a \in \Lambda^{+} \mid w \in E_{a}\right\}$, where we adopt the usual convention of taking $\max \emptyset=0$. In other words, we define $\pi$ in such a way that each $a$-cut $\pi_{a}$ coincides with $E_{a}$.

Note that, since by axioms (D) and (Nec) we have $v\left(\diamond_{1} \top\right)=1, E_{1} \neq \emptyset$. Then the last step is to show that, for every $\Psi \in \mathcal{L}_{\square}^{k+}, v(\Psi)=1$ iff $(w, \pi) \models$ $\Psi$.

We prove this by induction. The case $\Psi$ being a non-modal formula from $\mathcal{L}$ is clear, since in that case $w(\Psi)=v(\Psi)$. The interesting case is when $\Psi=\square_{a} \psi$. Then we have:

(i) If $v\left(\square_{a} \psi\right)=1$ then, by definition of $E_{1-p(a)}, E_{1-p(a)} \subseteq \operatorname{Mod}(\psi)$, and hence $(w, \pi) \models \square_{a} \psi$.

(ii) Conversely, if $E_{1-p(a)} \subseteq \operatorname{Mod}(\psi)$, then there must exist $\gamma$ such that $v\left(\square_{a} \gamma\right)=1$ and $\operatorname{Mod}(\gamma) \subseteq \operatorname{Mod}(\psi)$. Hence this means that $\gamma \rightarrow \psi$ is a PL theorem, and hence we have first, by the necessitation axiom, that $v\left(\square_{a}(\gamma \rightarrow \psi)\right)=1$, and thus $v\left(\square_{a} \gamma\right) \leq v\left(\square_{a} \psi\right)$ holds as well by axiom $(\mathrm{K})$, and therefore $v\left(\square_{a} \psi\right)=1$ holds as well.

As a consequence, we have that $(w, \pi) \models \Psi$ for all $\Psi \in \Gamma$ but $(w, \pi) \not \models \Phi$.

Similar to the non graded case of $\mathrm{MEL}^{+}$, we may consider an S5-like extension of $\mathrm{GPL}^{+}$, capturing the pointed possibilistic epistemic models $(w, \pi)$, where the 'actual world' $w$ is one of the non-discarded possible worlds by $\pi$. In this case, the higher $\pi(w)$ is, the more the actual world $w$ belongs to the set of plausible worlds, and hence we can speak of a notion of graded reflexive pointed possibilistic epistemic models $(w, \pi)$.

Definition 1 Let $(w, \pi)$ be a pointed possibilistic structure and let $a \in \Lambda^{+}$. We call $(w, \pi)$ to be a-reflexive when $\pi(w) \geq a$.

Let us define $\mathrm{GPL}_{a}^{++}$to be the axiomatic extension of $\mathrm{GPL}^{+}$with the following generalized $(\mathrm{T})$ axiom:

$\left(\mathrm{T}_{a}\right) \square_{a} \varphi \rightarrow \varphi$

One can check that $\left(\mathrm{T}_{a}\right)$ is valid in all $b$-reflexive pointed possibilistic structures, with $b=1-p(a)$. Indeed, if $(w, \pi) \models \square_{a} \varphi$ then $N_{\pi}(\varphi) \geq a$, and thus $\pi_{1-p(a)} \subseteq \operatorname{Mod}(\varphi)$. But if $(w, \pi)$ is $b$-reflexive, we have $\pi(w) \geq 1-p(a)$, and hence $w \in \pi_{1-p(a)} \subseteq \operatorname{Mod}(\varphi)$. Therefore $(w, \pi) \models \varphi$ as well. 
Theorem $3 \mathrm{GPL}_{a}^{++}$is sound and complete with respect to the class of (1$p(a))$-reflexive pointed possibilistic structures.

Proof: The proof is analogous to that of Theorem 2.

It is interesting to point out that Liau \& Lin $(1992,1993 \mathrm{a})$ have proposed a language similar to $\mathrm{GPL}^{+}$a long time ago, albeit using $[0,1]$ as a possibility scale (which forces them to introduce additional multimodal formulas to deal with strict inequalities) and graded accessibility relations. Their tableaubased proof methods could be of interest to develop inference techniques for GPL.

\section{Concluding remarks}

In this paper, we highlight the point that the fragment $\mathrm{MEL}^{+}$(resp. $\mathrm{MEL}^{++}$), involving modal formulas of depth 0 or 1, of the KD45 (resp. S5) logic, the richest among doxastic (resp. epistemic) logics, can have simplified semantics. Then we show that this result extends to graded modalities using the KD45 (resp. S5) extension $\mathrm{GPL}^{+}$(resp. $\mathrm{GPL}^{++}$) of the generalized possibilistic logic GPL. It makes it clear that the logic of incomplete information represented by possibility distributions is a fragment of known modal logics that do not request the use of full-fledged accessibility relations.

Besides, it has been recently shown that the graded notion of guaranteed possibility can be expressed in GPL enabling us to express "all I know" statements (Dubois et al., 2014) (see also (Banerjee \& Dubois, 2014) for the crisp case). This result calls for for a deeper comparison with the modal logic presented in (Dubois et al., 2000) that involves the classical modalities of the possible and the necessary together with the nonstandard modalities that are the guaranteed possibility and its dual, having also in mind that these four modalities and their negations make a cube of opposition (Ciucci et al., 2015a) that generalizes the square of opposition.

Finally, as the original intuition behind MEL is based on a situation where one agent reasons about the epistemic state of another agent, it would be natural to extend the possibilistic framework to the multiagent setting of reasoning about knowledge and cognitive planning.

\section{Dedication}

This article is particularly dedicated to Luis Fariñas del Cerro. It perfectly illustrates one of the topics at the junction of our respective subjects 
of interest, namely modal logic and possibility theory. Discussions along 35 years of friendship have repeatedly triggered two of the authors to dig more and more about the relations between these two knowledge representation frameworks, thanks also to the help of the two other authors of this note. Interestingly enough, while gaining mutual understanding of our respective reference theories, each of us has remained a supporter of one's own theory. Let us hope that in the long range, the now obvious bridge between the two formalisms will become routine knowledge so that both can be used appropriately by the same people according to the particulars of the applications at hand.

\section{Acknowledgements}

Godo is supported by the MINEC/FEDER project RASO (TIN2015-71799C2-1-P).

\section{References}

Banerjee, M., Dubois, D. (2009). A simple modal logic for reasoning about revealed beliefs. In C. Sossai and G. Chemelo (Eds.), Proc. 10th Europ. Conf. on Symbolic and Quantitative Approaches to Reasoning with Uncertainty (ECSQARU'09) (pp. 805-816). LNAI 5590, Springer.

Banerjee, M., Dubois, D., \& Godo L. (2014). Possibilistic vs. relational semantics for logics of incomplete information. In A. Laurent, O. Strauss, B. Bouchon-Meunier, R. R. Yager (Eds.) Proc. 15th Int. Conf. on Information Processing and Management of Uncertainty in Knowledge-Based Systems (IPMU'14), Part I (pp. 335-344), CCIS 442, Springer.

Banerjee, M., Dubois, D. (2014). A simple logic for reasoning about incomplete knowledge Int. J. Approximate Reasoning, 55(2), 639-653.

Béziau, J. Y. (2003). New light on the square of oppositions and its nameless corner. Logical Investigations, 10, 218-233.

Boutilier, C. (1994). Modal logics for qualitative possibility theory, Int. J. Approximate Reasoning, 10, 173-201.

Chellas, B. F. (1980). Modal Logic: an Introduction. Cambridge University Press. 
Ciucci, D., Dubois, D. (2014). Three-valued logics, uncertainty management and rough sets. Transactions on Rough Sets, Vol. XVII (pp.1-32), LNCS, Springer.

Ciucci, D., Dubois, D., \& Prade, H. (2014). The structure of oppositions in rough set theory and formal concept analysis - Toward a new bridge between the two settings. In: C. Beierle, C. Meghini (Eds.) Proc. 8th Int. Symp. on Foundations of Information and Knowledge Systems (FoIKS'14) (pp.154-173) LNCS 8367, Springer.

Ciucci, D., Dubois, D., \& Prade, H. (2015). Structures of opposition in fuzzy rough sets. Fundamenta Informaticae, 142, 1-19.

Ciucci, D., Dubois, D., \& Prade, H. (2016). Structure of opposition induced by relations. The Boolean and the gradual cases. Annals of Mathematics and Artificial Intelligence, 76(3), 351-373.

Dubois, D. (1986). Belief structures, possibility theory and decomposable confidence measures on finite sets. Computers and Artificial Intelligence (Bratislava), 5(5), 403-416.

Dubois, D., Hájek, P. \& Prade, H.(2000). Knowledge-driven versus datadriven logics. J. of Logic, Language, and Information, 9, 65-89.

Dubois, D., Lang, J., \& Prade, H. (1994). Possibilistic logic. In: D. M. Gabbay, C. J. Hogger, J. A. Robinson, D. Nute (Eds.) Handbook of Logic in Artificial Intelligence and Logic Programming, Vol. 3 (pp. 439-513) Oxford University Press.

Dubois, D., \& Prade, H. (1980) Fuzzy Sets and Systems: Theory and Applications, Mathematics in Science and Engineering Series, Vol. 144, Academic Press, New York.

Dubois, D., \& Prade, H. (1987). Twofold fuzzy sets and rough sets - Some issues in knowledge representation. Fuzzy Sets and Systems, 23 (1), 3-18.

Dubois, D., \& Prade, H. (1988). Possibility Theory: An Approach to Computerized Processing of Uncertainty, Plenum Press.

Dubois, D., \& Prade, H. (1990). Rough fuzzy sets and fuzzy rough sets, Int. J. of General Systems, 17(2-3), 191-209.

Dubois, D., \& Prade, H. (1990). Resolution principles in possibilistic logic. Int. J. of Approximate Reasoning, 4 (1), 1-21. 
Dubois, D., Prade, H. (2004). Possibilistic logic: a retrospective and prospective view. Fuzzy Sets and Systems, 144, 3-23.

Dubois, D., Prade, H. (2007). Toward multiple-agent extensions of possibilistic logic. Proc. IEEE Int. Conf. on Fuzzy Systems (FUZZ-IEEE'07), London, (pp. 187-192) IEEE.

Dubois, D., \& Prade, H. (2012). From Blanché's hexagonal organization of concepts to formal concept analysis and possibility theory. Logica Universalis, 6, 149-169,.

Dubois, D., \& Prade, H. (2014). Possibilistic logic - An overview. In: D. Gabbay, J. Siekmann, J. Woods (Eds.) Handbook of the History of Logic, vol. 9: Computational Logic (pp. 283-342) Elsevier.

Dubois, D., Prade, H. \& Rico, A. (2015). The cube of opposition: A structure underlying many knowledge representation formalisms. Proc. 24th Int. Joint Conf. on Artificial Intelligence (IJCAI'15), Buenos Aires, July 2531 (Q. Yang, M. Wooldridge, eds.), AAAI Press, 2933-2939.

Dubois, D., Prade, H. \& Schockaert, S. (2012). Stable models in generalized possibilistic logic. In: G. Brewka, T. Eiter, S. A. McIlraith (Eds.) Proc.13th Inter. Conf. on Principles of Knowledge Representation and Reasoning (KR'12) (pp. 519-529), AAAI Press.

Dubois, D., Prade, H. \& Schockaert, S. (2014). Reasoning about uncertainty and explicit ignorance in generalized possibilistic logic. In: T. Schaub, G. Friedrich, B. O'Sullivan (Eds.) Proc. 21st Europ. Conf. on Artificial Intelligence (ECAI'14) (pp. 261-266) IOS Press.

Dubois, D., Prade, H. \& Testemale, C. (1988). In search of a modal system for possibility theory. Proc. 8th Europ. Conf. on Artificial Intelligence (ECAI'88), Munich, (pp. 501-506).

Fagin, R. \& Vardi, M. Y. (1985). An internal semantics for modal logic. Proc. 17th ACM Symposium on Theory of Computing (STOC'85), Providence, RI. (pp. 305-315).

Halpern, J. Y., Fagin, R., Moses, Y. \& Vardi, M. Y. (2003). Reasoning About Knowledge. MIT Press (Revised paperback edition).

Fariñas del Cerro, L. \& Herzig, A. (1991). A modal analysis of possibility theory. In: Jorrand Ph. and Kelemen J. (Eds.) Fundamentals of Artificial 
Intelligence Research (FAIR'91) (pp.11-18) LNCS 535, Springer Verlag, Berlin.

Fariñas del Cerro, L. \& Orłowska, E. (1985). DAL - A logic for data analysis. Theoretical Computer Science, 36, 251-264.

Fariñas del Cerro, L. \& Prade, H.(1986). Rough sets, twofold fuzzy sets and modal logic. Fuzziness in indiscernibility and partial information. In: A. Di Nola, A. G. S. Ventre (Eds.- The Mathematics of Fuzzy Systems, Vol. 88 in Interdisciplinary Systems Research series, (pp. 103-120), Verlag TÜV Rheinland.

Hájek, P. (1994). A qualitative fuzzy possibilistic logic, Int. J. of Approximate Reasoning, 12, 1-19.

Hájek, P., Harmancová, D., Esteva, F., Garcia, P. \& Godo, L. (1994). On modal logics for qualitative possibility in a fuzzy setting. In R. Lopez de Mántaras and D. Poole (Eds.) Proc. 10th Conf. on Uncertainty in Artificial Intelligence (UAI'94) (pp. 278-285) Morgan Kaufmann.

Harmanec, D., \& Klir, G. J. (1994). On modal logic interpretation of Dempster-Shafer theory of evidence. Int. J. of Intelligent Systems, 9, 941951.

Harmanec, D., \& Klir, G. J. (1994). On modal logic interpretation of possibility theory. Int. J. of Uncertainty, Fuzziness and Knowledge-based Systems, 2 (2), 237-245.

Hendricks, V., \& Symons, J. (2015). Epistemic logic. In E. N. Zalta (Ed.) The Stanford Encyclopedia of Philosophy (Fall 2015 Edition), URL = https://plato.stanford.edu/archives/fall2015/entries/logic-epistemic.

Lakoff, G. (1973). Hedges: A study in meaning criteria and the logic of fuzzy concepts. J. of Philosophical Logic, 2, 458-508.

Lang, J., Dubois, D., \& Prade, H. (1991). A logic of graded possibility and certainty coping with partial inconsistency. Proc. 7th Conf. on Uncertainty in Artificial Intelligence (UAI'91) (pp. 188-196) Morgan-Kaufmann, Palo Alto, Ca.

Lewis, D. (1973) Counterfactuals and comparative possibility. Journal of Philosophical Logic, 2 (4), 418-446.

Lewis, D. (1973). Counterfactuals. Oxford, UK, Basil Blackwell. 
Liau, C.-J., \& Fan, T.-F. (2005). A modal logic for reasoning about possibilistic belief fusion. In: L. Pack Kaelbling, A. Saffiotti (Eds.) Proc. 19th Int. Joint Conf. on Artificial Intelligence (IJCAI'05), Edinburgh, 17561757 .

Liau, C.-J., \& Lin, B. I-Peng. (1992). Quantitative modal logic and possibilistic reasoning. In: B. Neumann (Ed.) Proc. 10th Europ. Conf. on Artificial Intelligence (ECAI'92), (pp. 43-47) John Wiley and Sons, Chichester.

Liau, C.-J., \& Lin, B. I-Peng. (1993) Proof methods for reasoning about possibility and necessity. Int. J. Approx. Reasoning, 9 (4), 327-364.

Liau, C.-J., \& Lin, B. I-Peng. (1993) Reasoning about higher order uncertainty in possiblistic logic. In: H. J. Komorowski, Z. W. Ras (Eds.) Proc. 7th Int. Symp. on Methodologies for Intelligent Systems (ISMIS'93) (pp. 316-325) LNCS 689, Springer.

Lipski, W. (1981). On databases with incomplete information. J. of ACM, $28(1), 41-70$.

Orłowska, E. (1985). A logic of indiscernibility relations. In: Computation Theory (pp. 177-186) LNCS 208, Springer-Verlag, Berlin.

Pawlak, Z. (1991) Rough Sets. Theoretical Aspects of Reasoning about Data, Kluwer.

Pawlak, Z., \& Skowron, A. (2007). Rudiments of rough sets. Information Sciences 177, 3-27.

Petruszczak, A. (2009). Simplified Kripke style semantics for modal logics K5, KB5 and KD45. Bulletin of the Section of Logic 38, 163-171.

Prade, H. (1985). Reasoning with fuzzy default values. Proc. 15th IEEE Inter. Symp. Multiple-Valued Logic, Kingston, On., Canada, (pp. 191-197) IEEE.

Resconi, G., Klir, G. J., \& St. Clair, U. (1992). Hierarchical uncertainty metatheory based upon modal logic. Int. J. General Systems, 21, 23-50.

Resconi, G., Klir, G. J., Harmanec, D. \& St. Clair, U. (1996). Interpretations of various uncertainty theories using models of modal logic: a summary. Fuzzy sets and Systems, 80, 7-14. 
Resconi, G., Klir, G. J., St. Clair, U. \& Harmanec, D. (1993). On the integration of uncertainty theories. Int. J. of Uncertainty, Fuzziness and Knowledge-based Systems, 1, 1-18.

Schotch, P. K. (1975) Fuzzy modal logic. Proc. 5th IEEE Int. Symp. on Multiple-Valued Logic, Bloomington, IN, (pp. 176-182) IEEE.

Shackle, G. L. S. (1961). Decision, Order, and Time in Human Affairs. Cambridge Univ. Press.

Thiele, H. (1998). Fuzzy Rough Sets versus Rough Fuzzy Sets. An Interpretation and a Comparative Study using Concepts of Modal Logics. Research report No. CI-30/ 98, University of Dortmund, Germany.

Touazi, F., Cayrol, C., \& Dubois, D. (2015). Possibilistic reasoning with partially ordered beliefs. J. Applied Logic 13(4): 770-798.

Yao, Y. \& Lin, T. (1996). Generalization of rough sets using modal logic. Automation and Soft Computing 2(2), 103-120.

Yao, Y., Wang S., \& Lin, T. (1997). A review of rough set models. In: L. Polkowski, A. Skowron (Eds.) Rough Sets and Data Mining: Analysis for Imprecise Data (pp. 47-75) Kluwer Acad. Publ.

Zadeh, L. A. (1978). Fuzzy sets as a basis for a theory of possibility. Fuzzy Sets and Systems 1, 3-28.

Zadeh, L. A. (1978). PRUF - A meaning representation language for natural languages. Int. J. Man-Machine Studies 10, 395-460.

Zadeh, L. A. (1979). A theory of approximate reasoning. In: J. E. Hayes, D. Mitchie, L. I. Mikulich (Eds.) Machine Intelligence, vol. 9, (pp. 149-194) Ellis Horwood.

Zadeh, L. A. (1979) Fuzzy sets and information granularity. In: M. M. Gupta, R. K. Ragade, R. R. Yager, (Eds.) Advances in Fuzzy set Theory and Applications (pp. 3-18) North-Holland. 